\title{
Interstitial lactate, lactate/pyruvate and glucose in rat muscle before, during and in the recovery from global hypoxia
}

\author{
Norbert Zoremba ${ }^{1 *}$, Aleš Homola ${ }^{2,3}$, Rolf Rossaint ${ }^{1}$ and Eva Syková ${ }^{2,3}$
}

\begin{abstract}
Background: Hypoxia results in an imbalance between oxygen supply and oxygen consumption. This study utilized microdialysis to monitor changes in the energy-related metabolites lactate, pyruvate and glucose in rat muscle before, during and after 30 minutes of transient global hypoxia. Hypoxia was induced in anaesthetised rats by reducing inspired oxygen to $6 \% \mathrm{O}_{2}$ in nitrogen.

Results: Basal values for lactate, the lactate/pyruvate ratio and glucose were $0.72 \pm 0.04 \mathrm{mmol} / \mathrm{l}, 10.03 \pm 1.16$ and $3.55 \pm 0.19 \mathrm{mmol} / \mathrm{l}(\mathrm{n}=10)$, respectively. Significant increases in lactate and the lactate/pyruvate ratio were found in the muscle after the induction of hypoxia. Maximum values of $2.26 \pm 0.37 \mathrm{mmol} / \mathrm{l}$ for lactate were reached during early reperfusion, while the lactate/pyruvate ratio reached maximum values of $35.84 \pm 7.81$ at the end of hypoxia. Following recovery to ventilation with air, extracellular lactate levels and the lactate/pyruvate ratio returned to control levels within 30-40 minutes. Extracellular glucose levels showed no significant difference between hypoxia and control experiments.
\end{abstract}

Conclusions: In our study, the complete post-hypoxic recovery of metabolite levels suggests that metabolic enzymes of the skeletal muscle and their related cellular components may be able to tolerate severe hypoxic periods without prolonged damage. The consumption of glucose in the muscle in relation to its delivery seems to be unaffected.

Keywords: Hypoxia, Microdialysis, Muscle, Recovery, Lactate, Glucose, Metabolism

\section{Background}

The skeletal muscles are an elaborate energy production and consumption system that influences the whole body's energy metabolism [1]. The metabolism of skeletal muscle is strictly regulated by substrate availability, the presence of oxygen and energy demands, which in turn also regulate muscle protein metabolism and cell size [2]. Hypoxia causes a deficiency in cellular oxygen supply and may lead to tissue damage, an inflammatory response and organ dysfunction [3]. This imbalance between oxygen consumption and supply initiates changes in extracellular metabolite and substrate levels, due to their release or uptake by cells to and from the extracellular space (ECS). Microdialysis is a highly sensitive technique used to monitor extracellular metabolite and substrate levels

\footnotetext{
* Correspondence: nzoremba@ukaachen.de

'Department of Anaesthesiology, University Hospital RWTH Aachen,

Pauwelsstrasse 30, D-52074 Aachen, Germany

Full list of author information is available at the end of the article
}

and allows the measurement of regional metabolic tissue concentrations [4].

In clinical practice lactate has been interpreted as a marker of anaerobic metabolism, if an imbalance between tissue $\mathrm{O}_{2}$ supply and demand occurs. Lactate accumulates as the end product of glycolysis when oxidative phoshorylation, as well as the tricarboxylic acid cycle are reduced [5-8]. The isolated view of lactate as a marker for anaerobic metabolism could be misleading. For the detection of anaerobic metabolism, the simultaneous determination of pyruvate levels is necessary, as pyruvate is reduced to lactate by lactate-dehydrogenase under anaerobic conditions. By examining the ratio between lactate and pyruvate ( $\mathrm{L} / \mathrm{P}$ ratio) the degree of aerobic/anaerobic metabolism can be specified, reflecting the cytosolic ratio of the reduced/oxidized forms of NAD. Therefore, the L/P ratio is a more reliable parameter for estimating the energy state of the cells [8]. In addition to the measurement of metabolic end products, the extracellular glucose level is 
an important factor in substrate equilibration. The glucose concentration in the ECS reflects the balance between the supply from blood and utilisation by cells [9].

The aim of our study was to evaluate the changes in the energy-related metabolites lactate and pyruvate as well as in the substrate glucose before, during and after global hypoxia in the skeletal muscle using in vivo microdialysis. These findings may help to describe the effects of hypoxia and reoxygenation on muscular aerobic and anaerobic metabolism and substrate levels.

\section{Methods}

\section{Animal preparation and experimental protocol}

Adult male Wistar rats (300-350 g and aged approximately 12-15 weeks), obtained from the same breeder (Velaz, s.r. o, Prague, Czech Republic), were housed in groups of 4 animals per cage before the experiment in breeding cages T IV (Velaz, s.r.o, Prague, Czech Republic). The cages was provided with spruce bedding (Lignocel ${ }^{\circledR} 3-4 S$, Rettenmaier und Söhne, Rosenberg, Germany), and no further enrichment were provided. The rats were housed in a 12:12 hour light:dark cycle with lights turning on at 6 o'clock in the morning. The room temperature was maintained at $22^{\circ} \mathrm{C}$. Food (Altromin 1314 TPF standard diet, Altromin Spezialfutter $\mathrm{GmbH}$ \& Co KG, Lage, Germany) and tapwater was provided ad libitum. The animals were anaesthetised by an intraperitoneal injection of urethane (1.5 g/kg body weight, Sigma-Aldrich Chemie $\mathrm{GmbH}$, Seelze, Germany). Before the beginning of the surgical procedures, the depth of anaesthesia was checked by testing the corneal reflexes. If necessary, an additional $100 \mathrm{mg}$ of urethane were injected intraperitoneally. To avoid any ventilatory interactions due to spontaneous breathing action, the animals were relaxed with pancuroniumbromide $(0.4 \mathrm{mg} / \mathrm{kg}$ body weight, Pavulon, Organon, Netherlands), intubated and connected to a ventilator (CIV 101, Columbus Instruments, Columbus, Ohio, USA). The body temperature was maintained at $36-37^{\circ} \mathrm{C}$ by a heating pad. The sufficient depth of anaesthesia during the experiment was monitored by means of clinical signs (heart rate, lacrimation etc.). A small longitudinal incision of the skin was made above the back muscles to place the microdialysis probe. This device was slowly inserted into the musculus longissimus by an introducing canula. To avoid any possible influence of probe insertion tissue trauma on the microdialysis results, measurements started one hour after the placement of the microdialysis probe. Before hypoxia the animals were ventilated with air. Hypoxia was induced for 30 minutes by reducing the inspiratory oxygen content to $6 \%$ in $94 \%$ nitrogen. After this hypoxic period the animals were ventilated with air again. The control animals were ventilated with air during the whole experiment. This study was performed on hypoxic $(n=10)$ and control animals $(n=5)$, which were randomly assigned to the two groups before starting the experiment. At the end of the experiment the animals were euthanized by intraperitoneal injection of urethane and potassium chloride.

All efforts were made to minimize animal suffering and to reduce the number of animals used. The experiments were carried out in accordance with the European Communities Council Directive of $24^{\text {th }}$ November 1986 (86/609/EEC) and approved by the local Institutional Animal Ethics Committee.

\section{Microdialysis}

The measurement of extracellular metabolite and substrates levels by microdialysis is based on sampling fluid via a double-lumen probe with an integrated semipermeable membrane. At this membrane an equilibration of substances in the extracellular space and the perfusion fluid takes place by diffusion according to the concentration gradient. We used a double-lumen microdialysis probe with a membrane length of $10 \mathrm{~mm}$, an outer diameter of $0.5 \mathrm{~mm}$ and a cut-off at 20.000 Dalton (CMA 20, CMA Microdialysis, Sweden). In order to maintain a constant dialysate flow, the microdialysis catheter was connected via low-volume FEP-tubing $(1.2 \mu \mathrm{l} / 10 \mathrm{~cm})$ to a precision infusion pump (CMA 102, CMA Microdialysis, Sweden). The microdialysis catheter was continuously perfused with a dialysate containing $147 \mathrm{mmol} / \mathrm{l} \mathrm{Na}^{+}, 4.0 \mathrm{mmol} / \mathrm{l} \mathrm{K}^{+}$, $2.3 \mathrm{mmol} / 1 \mathrm{Ca}^{2+}$ and $155.6 \mathrm{mmol} / \mathrm{l} \mathrm{Cl}^{-}$(Perfusion fluid T1, CMA Microdialysis, Sweden) at a flow rate of $2 \mu \mathrm{l} / \mathrm{min}$. It has been shown that probe insertion damage causes a transient increase in metabolite levels, which return to stable baseline values within a stabilisation period of 30-60 minutes after probe insertation [10]. After an equilibration time of 60 minutes, microdialysate samples were collected at 10 minute intervals and immediately frozen at $-40^{\circ} \mathrm{C}$ until analyzed. Dialysate samples were analysed enzymatically with a CMA 600 Microdialysis Analyser (CMA Microdialysis, Sweden) for lactate, pyruvate and glucose.

The exchange of substances across the microdialysis membrane is limited by the total area of the membrane, the perfusion flow rate, the characteristics of the diffusing substance and the diffusion constant in the tissue surrounding the probe $[4,11]$. These boundaries are described by the recovery rate, which expresses the relation between the concentration of the substance in the microdialysis probe effluent and the concentration of the medium [12]. At the beginning and end of the experiment, the recovery rates for each probe were determined by proceeding with the perfusion in the same settings in a defined calibration solution. The calibration solution contained $2.50 \mathrm{mmol} / \mathrm{l}$ lactate, $250 \mu \mathrm{mol} / \mathrm{l}$ pyruvate and $5.55 \mathrm{mmol} / \mathrm{l}$ glucose (Calibrator A, CMA Microdialysis, Sweden). The concentrations of these calibration solutions were compared 
with the concentrations of the in vitro microdialysis samples, and the relative recovery for each substance was calculated. The measured experimental values were weighted by the relative recovery to estimate the in vivo extracellular concentration of the components in the immediate vicinity of the probes. All results are presented as weighted concentrations.

\section{Statistical analysis}

The results of the experiments are expressed as the mean \pm standard error of the mean (SEM). Statistical analysis of the differences within and between groups was performed using Student's paired t-test (InStat, GraphPad Software, San Diego, USA). Values of $P<0.05$ were considered significant. If a statistical significant difference was found a post hoc power analysis was made with $\alpha=0.05$.

\section{Results}

Lactate and lactate/pyruvate ratio

Before hypoxia, the basal muscular lactate levels remained stable at $0.72 \pm 0.04 \mathrm{mmol} / \mathrm{l}(\mathrm{n}=10)$. Immediately after the onset of hypoxia $\left(6 \% \mathrm{O}_{2}\right)$ a steep increase in extracellular lactate was measured. This rise continued throughout the hypoxic period of 30 minutes, reaching maximum values of $2.26 \pm 0.37 \mathrm{mmol} / \mathrm{l}$ in the first measurement after the end of hypoxia. During reoxygenation with air, a recovery of the lactate level occurred, returning to the basal level within 40 minutes. In the control animals a basal lactate value of $0.73 \pm 0.03 \mathrm{mmol} / \mathrm{l}(\mathrm{n}=5)$ was measured, which showed no significant changes during the measurement period. The difference between control and hypoxic animals was statistical significant $(P<0.05)$ during hypoxia and early recovery (Figure $1 \mathrm{~A}$ ). In a post hoc power analysis a statistical power $>80 \%$ was calculated. To recognize the switch to an anaerobic pathway, we calculated the lactate/pyruvate ratio (L/P ratio). In animals subjected to hypoxia a significant increase in the $\mathrm{L} / \mathrm{P}$ ratio was seen from $10.03 \pm 1.16$ during baseline registration to $35.84 \pm 7.81(n=10)$ at the end of the hypoxic period (Figure 1B). These changes in the $\mathrm{L} / \mathrm{P}$ ratio produced a statistical power $>80 \%$. In the reoxygenation period the $\mathrm{L} / \mathrm{P}$ ratio normalised, reaching baseline levels of $11.01 \pm 0.88$ within 30 minutes and remaining at this level until the end of the measurement period. In the control animals a basal L/P ratio of $8.22 \pm$ $0.81(\mathrm{n}=5)$ was registered. There were no significant changes in the L/P observed during the entire observation period.

\section{Glucose}

Before the induction of hypoxia, stable basal glucose levels of $3.55 \pm 0.19 \mathrm{mmol} / \mathrm{l}(\mathrm{n}=10)$ were found in the hypoxia group and $3.81 \pm 0.22 \mathrm{mmol} / \mathrm{l}(\mathrm{n}=5)$ in the control group. During the whole experiment no significant differences
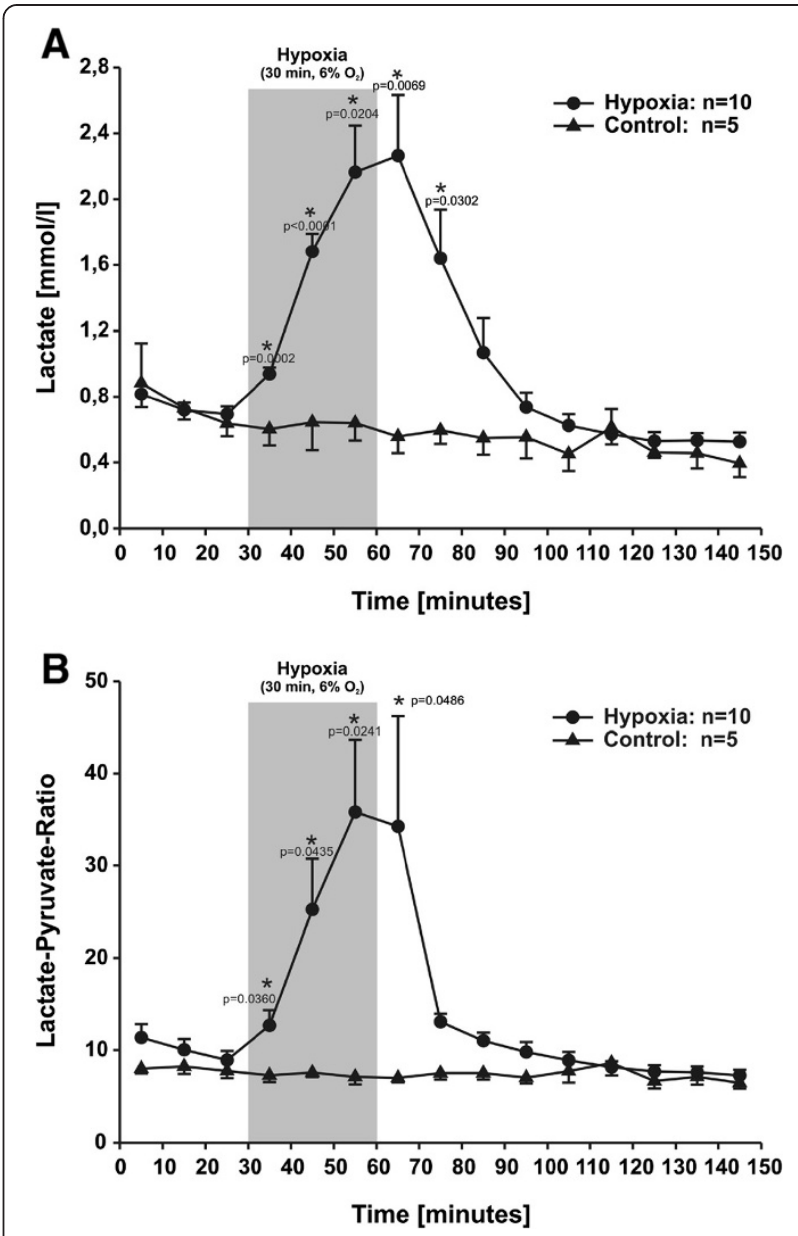

Figure 1 Interstitial lactate levels (A) and the L/P ratio (B) before, during and after $\mathbf{3 0}$ minutes of hypoxia compared to controls.

After a stabilisation period, hypoxia was induced for 30 minutes by ventilating the animals with $6 \%$ oxygen in nitrogen (shaded area). Values are shown as mean \pm SEM. The number of animals was $n=10$ in the experimental and $n=5$ in the control group. Statistically significant differences between the experimental and control groups were determined by Student's t-test. Values of $P<0.05$ are marked by "**".

were found between the two groups. At the end of the experiment a glucose level of $2.96 \pm 0.27 \mathrm{mmol} / \mathrm{l}$ was measured in the hypoxia group. In the control group the final glucose level was $2.89 \pm 0.25 \mathrm{mmol} / \mathrm{l}$. The time course of the extracellular glucose levels in both groups is shown in Figure 2.

\section{Discussion}

The aim of this study was to evaluate the changes in extracellular muscular lactate, the lactate/pyruvate $(\mathrm{L} / \mathrm{P})$ ratio and glucose levels during a hypoxic $\left(6 \% \mathrm{O}_{2}\right)$ period of 30 minutes duration and subsequent reoxygenation. Microdialysis enables obtaining local information on energy metabolism in vivo in any tissue by introducing a probe directly into the region of interest [10]. An inspiratory oxygen content of $6 \%$ is the lowest possible level that 


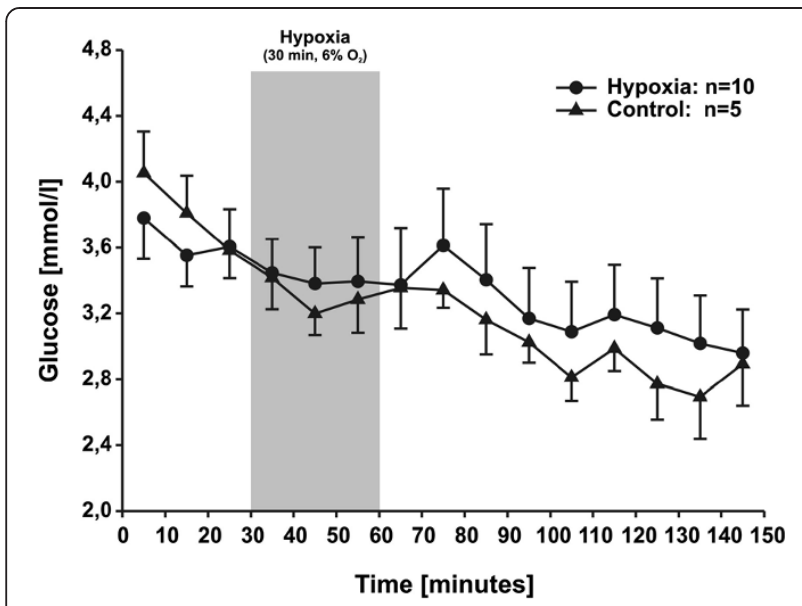

Figure 2 Interstitial muscular glucose levels before, during and after $\mathbf{3 0}$ minutes of hypoxia compared to controls. The duration of hypoxia is marked by a shaded area. Values are shown as mean \pm SEM, and the number of animals was $n=10$ in the hypoxic and $n=5$ in the control measurements. No statistically significant differences were found between the groups.

can be used for this experimental model, as lower levels result in cardiac arrhythmia and cardiac failure. To the best of our knowledge no other evaluations of lactate, lactate/pyruvate ratio and glucose in the muscle were undertaken in an experimental setting with such a low hypoxic level. The possible influences of other organs were taken into consideration by choosing the rat as the experimental model animal.

In our experiments the onset of hypoxia was accompanied by a significant increase in muscular lactate levels and the L/P ratio. This increase in lactate levels and the lactate/pyruvate ratio is similar to changes observed in muscle that were reported in previous studies during hypoxia with $10 \% \mathrm{O}_{2}[13,14]$. Hypoxia leads to an increase in production of lactic acid due to a switch from aerobic to anaerobic metabolism. In aqueous solutions lactic acid dissociates almost completely to lactate and $\mathrm{H}^{+}$ [15]. An increase has also been found in different tissues as well as in the blood, accompanied by severe acidosis $[14,16]$. Severe acidosis may lead to possible disturbances in intra/extracellular ion distribution. The liberation of lactate may be much faster than its removal due to sufficient circulation. Additionally this increase in lactate level can be worsened by decreasing, hypoxia-induced hepatic lactate clearance [15]. Following hypoxia a fall in cellular ATP stimulates the activity of phosphofructokinase and an increase in glycolysis occurs. Nicotinamide adenine dinucleotide $\left(\mathrm{NAD}^{+}\right)$, from the conversion of pyruvate to lactate, is required to support this increase in glycolysis [15]. Furthermore, an increase of glycolysis can be additionally amplified by endogenous secretion of catecholamines, due to stress during hypoxia.
During the reoxygenation period the elevated lactate levels recovered to prehypoxic values. This normalisation of extracellular muscular lactate levels could be caused by its release into the blood and consumption by the liver or by its uptake and consumption in the muscle. It was shown that blood levels during hypoxia are much higher than in the muscle and therefore the skeletal muscles act as lactate consumers even in the hypoxic state [13]. Muscles are also able to oxidize lactate, especially in oxidative fibers $[17,18]$. Thus, it could be hypothesized that during reoxygenation, the interstitial lactate was at least in part consumed in the muscle. In addition to the lactate level, the tissue $\mathrm{L} / \mathrm{P}$ ratio is an excellent marker of anaerobic metabolism as it correlated closely with the redox potential [8]. In our study we found a steep increase in the L/P ratio starting immediately after the onset of hypoxia. This increase reflects the switch of the cytosolic redox condition from aerobic to anaerobic glycolysis. In the recovery from hypoxia a steep decrease in the $L / P$ ratio indicates the return to aerobic conditions. These results indicate that energy-related cellular components (e.g. mitochondria) and their related metabolic enzymes may tolerate such hypoxic periods without any serious damage.

The extracellular glucose level is maintained by a balance between supply from the blood and utilisation by cells. During hypoxia no significant difference in muscular glucose levels between the hypoxia and control groups was found. It has been shown that during hypoxia the mean arterial blood pressure decreases about $50 \%$ from the prehypoxic values [14]. In the cerebral cortex, a tissue with high metabolism, reduction in arterial perfusion pressure during hypoxia results in a decrease in glucose levels and normalisation during the recovery [16]. It could be hypothesized that in skeletal muscle, despite a reduction in systemic blood pressure, a sufficient glucose supply is present. Therefore, the extracellular glucose concentration remained unaffected at the prehypoxic levels.

\section{Methodological considerations}

In our experiments we used the microdialysis technique for determining regional metabolic tissue concentrations. This method is highly sensitive, but has some methodical limitations that must be addressed. The in vivo recovery of substances strongly depends on the surrounding tissue properties and can be influenced by various release, uptake and clearance processes [19]. The calculations of extracellular metabolite concentrations based on in vitro recovery may underestimate the interstitial concentrations [19-21]. However, the collected experimental data reflect the dynamic time course of extracellular microdialysate levels during and after hypoxia. These experiments represent the evaluation of energy-related metabolites and glucose levels during the lowest possible hypoxia levels in a full animal model. Further experiments with longer 
hypoxic periods are needed to describe in more detail the behaviour of skeletal muscles during and after hypoxic conditions.

\section{Conclusion}

In conclusion, our findings suggest that 30 minutes of hypoxia influences the extracellular microenvironment of skeletal muscles and that the changes in energy-related metabolites caused by hypoxia recover to preischemic values within a few minutes. The energy-related cellular components (e.g. mitochondria) and their related metabolic enzymes may tolerate such a hypoxic period without any serious or prolonged damage. The glucose levels seem to be unaffected during and after hypoxia. It could be, that despite a possible reduction in systemic blood pressure, hypoxia does not affect sufficient supply to skeletal muscle.

\section{Competing interests}

The authors declare that they have no competing interests.

\section{Authors' contributions}

$\mathrm{NZ}, \mathrm{AH}$ and ES conceived the study, participated in the study's design and coordination, performed the statistical analysis and drafted the manuscript. $\mathrm{NZ}$ and $\mathrm{AH}$ conducted the experimental laboratory work. RR assisted in drafting the manuscript. All authors read and approved the final manuscript.

\section{Acknowledgement}

This study was supported by the European Commission Marie Curie Training Site Programme HPMT-CT-2000-00187, by grant AV0Z50390512 from the Academy of Sciences of the Czech Republic and by grants 1M0021620803, LC544 and GACR 309/09/1597 from the Ministry of Sport, Youth and Education of the Czech Republic.

\section{Author details}

'Department of Anaesthesiology, University Hospital RWTH Aachen, Pauwelsstrasse 30, D-52074 Aachen, Germany. ${ }^{2}$ Department of Neuroscience, Charles University, 2nd Medical Faculty, V Úvalu 84, 15006 Prague, Czech Republic. ${ }^{3}$ Institute of Experimental Medicine, Academy of Sciences of Czech Republic, Vídeňská 1083, 14220 Prague, Czech Republic.

Received: 5 November 2013 Accepted: 15 October 2014

Published online: 13 November 2014

\section{References}

1. lizuka K, Machida T, Hirafuji M: Skeletal muscle is an endocrine organ. J Pharmacol Sci 2014, 125:1-7.

2. Koopman R, Ly CH, Ryall JG: A metabolic link to skeletal muscle wasting and regeneration. Front Physiol 2014, 5:32.

3. Willis N, Mogridge J: Indicators of histohypoxia. Acta Anaesthesio/ Scand Suppl 1995, 107:45-48.

4. Ungerstedt U: Microdialysis - principles and applications for studies in animal and man. J Intern Med 1991, 230:365-373.

5. De Salles AA, Kontos HA, Becker DP, Yang MS, Ward JD, Moulton R, Gruemer HD, Lutz H, Maset AL, Jenkins L: Prognostic significance of ventricular CSF lactic acidosis in severe head injury. J Neurosurg 1986, 65:615-624.

6. Inao S, Marmarou A, Clarke GD: Production and clearance of lactate from brain tissue, cerebrospinal fluid, and serum following experimental brain injury. J Neurosurg 1998, 69:736-744.

7. Mizock BA, Falk JL: Lactic acidosis in critical illness. Crit Care Med 1992 20:80-93.

8. Magnoni S, Ghisoni L, Locatelli M, Caimi M, Colombo A, Valeriani V, Stocchetti N: Lack of improvement in cerebral metabolism after hyperoxia in severe head injury: a microdialysis study. J Neurosurg 2003, 98:952-958.
9. Newman JM, Rattigan S, Clark MG: Nutritive blood flow improves interstitial glucose and lactate exchange in perfused rat hindlimb. Am J Heart Circ Physiol 2002, 283:186-192.

10. Valtysson J, Persson L, Hillered L: Extracellular ischemia markers in repeated global ischemia and secondary hypoxaemia monitored by microdialysis in rat brain. Acta Neurochir 1998, 140:387-395.

11. Arner P, Bolinder J: Microdialysis of adipose tissue. J Int Med 1991, 230:381-386.

12. Muller M: Science, medicine, and the future: microdialysis. Br Med J 2002, 324:588-591.

13. Fuse A: The role of skeletal muscle and liver on lactate metabolism during hypoxia in rats. J Anesth 1999, 13:161-165.

14. Klaus S, Heringlake M, Gliemroth J, Pagel H, Staubach K, Bahlmann L: Biochemical tissue monitoring during hypoxia and reoxygenation. Resuscitation 2003, 56:299-305.

15. Phypers B, Pierce T: Lactate physiology in health and disease. Contin EduC Anaesth Crit Care Pain 2006, 6:128-132.

16. Zoremba N, Homola A, Rossaint R, Sykova E: Brain metabolism and extracellular space diffusion parameters during and after transient global hypoxia in the rat cortex. Exp Neurol 2007, 203:34-41.

17. Pagliassotti MJ, Donovan CM: Role of cell type in net lactate removal by skeletal muscle. Am J Physiol 1990, 258:635-642.

18. Gladden LB: Net lactate uptake during progressive steady-level contractions in canine skeletal muscle. J Appl Physiol 1991, 71:514-520.

19. Benveniste $H$, Huttemeier PC: Microdialysis - Theory and application Prog Neurobiol 1990, 35:195-215.

20. Benveniste $H$, Hansen AJ, Ottosen NS: Determination of brain interstitial concentrations by microdialysis. J Neurochem 1989, 52:1741-1750.

21. Chen KC, Hoistad M, Kehr J, Fuxe K, Nicholson C: Theory relating in vitro and in vivo microdialysis with one or two probes. J Neurochem 2002, 81:108-121.

\section{doi:10.1186/s13028-014-0072-0}

Cite this article as: Zoremba et al:: Interstitial lactate, lactate/pyruvate and glucose in rat muscle before, during and in the recovery from global hypoxia. Acta Veterinaria Scandinavica 2014 56:72.

\section{Submit your next manuscript to BioMed Central and take full advantage of:}

- Convenient online submission

- Thorough peer review

- No space constraints or color figure charges

- Immediate publication on acceptance

- Inclusion in PubMed, CAS, Scopus and Google Scholar

- Research which is freely available for redistribution 